\title{
Statistics of orbital entanglement production in a chaotic quantum dot with nonideal contacts
}

\author{
Francisco A. G. Almeida and Andre M. C. Souza \\ Departamento de Fisica, Universidade Federal de Sergipe, 49100-000 Sao Cristovao, SE, Brazil \\ (Received 4 May 2010; revised manuscript received 12 August 2010; published 13 September 2010)
}

\begin{abstract}
We investigate the statistics of orbital entanglement production between electrons in a chaotic quantum dot with two-channel leads. Through a random-matrix simulation, we obtain the probability density of two entanglement measures, concurrence and entanglement formation, by varying the transparency of the contacts in the presence and absence of time-reversal invariance of the electron dynamics inside the cavity. The results suggest that orbital entanglement production is optimized by increasing the asymmetry between the transparency of the contacts, especially when time-reversal invariance is broken.
\end{abstract}

DOI: $10.1103 /$ PhysRevB.82.115422

PACS number(s): 73.23.-b, 03.67.Bg, 73.63.Kv, 05.45.Pq

\section{INTRODUCTION}

Quantum entanglement refers to nonlocal correlations between two or more particles and it cannot be obtained by means of classical mechanisms. ${ }^{1}$ This phenomenon has caused a conceptual challenge to the understanding of the foundations of quantum mechanics. Furthermore, the information science community has been interested in controlling the production of entangled states because it can be used to advance many technologies based on cryptography, teleportation, and computation. ${ }^{1,2}$

Since it is possible to produce entanglement between electrons, there are many efforts to study entanglement in solidstate systems. ${ }^{3-20}$ In particular, there are many quantum information applications for noninteracting electron systems. In this way, a chaotic quantum dot (see Fig. 1) was proposed as an orbital entangler between a noninteracting electron pair. 6,7

The chaotic quantum dot is fundamental to study theoretically ${ }^{21,22}$ and experimentally ${ }^{23,24}$ quantum transport in mesoscopic systems. It can be modeled as a chaotic cavity coupled to lead nonideally as illustrated by Fig. 1 . There is a bias voltage applied in the leads extremities which induces the electronic transport. Therefore, the quantum dot can be viewed as a scattering center where each lead has some open scattering channels and, furthermore, the electron dynamics into the cavity is chaotic.

Using a model of two-channel leads and neglecting the contact effects between leads and chaotic cavity, it was possible to study analytically the statistic of orbital entanglement production in a quantum dot in the presence and absence of time-reversal invariance (TRI). Initially, Beenakker et $a .^{6}{ }^{6}$ verified that the averages and variances of two entanglement measures (concurrence and entanglement formation) are weakly sensitive to TRI effects. Nevertheless, because the system has only two open scattering channels, it is in the extreme quantum limit where there are very irregular fluctuations of the observables. ${ }^{25}$ Thus, Gopar and Frusgtalia ${ }^{17}$ noticed that the averages and variances of these entanglement measures contain insufficient information and, therefore, they investigated the full probability densities to describe the entanglement statistics with fidelity. This careful statistical analysis showed that TRI effects induce different behaviors in entanglement production.
There are more features that can affect the statistics of entanglement production in a quantum dot, for example, contact effects. The transparency of the contact is equivalent to the probability of the electron tunneling through the contact and it can be controlled experimentally. ${ }^{23}$ Thus, there are two more system parameters (two contact transparencies) that can be manipulated in the system to modify the production of entanglement. The present work addresses this issue. We investigate the full statistics of orbital entanglement production by numerical simulation techniques ${ }^{25}$ in the presence and absence of TRI for various values of transparency of the contacts. Due to the interest in information technology, we are focused on the optimization of entanglement production.

This paper is organized as follows. In Sec. II we review the proposal of a quantum dot as an orbital entangler. The numerical method that we used to simulate the system is briefly described in Sec. III. The results of the statistics of orbital entanglement production are presented in Sec. IV in two parts: symmetric contacts in A and arbitrary ones in B. Finally, we present a summary and our conclusions in Sec. V.

\section{QUANTUM DOT AND ORBITAL ENTANGLEMENT}

We assume in the quantum dot model studied in this paper that transport is phase coherent, there are no electronelectron interactions and each lead has only two open scattering channels. In this situation, the $4 \times 4$ unitary scattering matrix of the system is

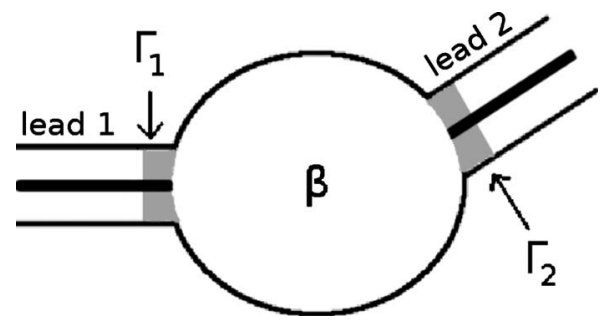

FIG. 1. Schematic view of a chaotic quantum dot with two double-channel leads. The solid line inside each lead represents an illustrative separation between the two open scattering channels. The transparency of the contact between lead $\nu$ and the chaotic cavity is $\Gamma_{\nu}$. The physical symmetry of electron dynamics inside the cavity is indexed by $\beta$. 


$$
\mathbf{S}=\left(\begin{array}{ll}
\mathbf{r} & \mathbf{t}^{\prime} \\
\mathbf{t} & \mathbf{r}^{\prime}
\end{array}\right),
$$

where $\mathbf{r}$ and $\mathbf{t}\left(\mathbf{r}^{\prime}\right.$ and $\left.\mathbf{t}^{\prime}\right)$ are the $2 \times 2$ blocks of reflection and transmission of the left-incident (right-incident) electrons. The scattering matrix relates the incoming amplitudes of the wave function to the outgoing ones and, in general, it has the essential feature of mixing the incoming amplitudes among all open scattering channels. Moreover, the electronic transport can be characterized by the transmission eigenvalues $\tau_{1}$ and $\tau_{2}$ of the Hermitian matrix $\mathbf{t t}^{\dagger} .{ }^{21,22}$

Let us give a brief explanation of how the system can be an orbital entangler based on Refs. 7, 6, and 17. Let us use the notation $\left|n_{11}, n_{12}, n_{21}, n_{22}\right\rangle$ for a two-electron state of the system, with $\Sigma_{\mu j} n_{\mu j}=2$, where $n_{\mu j}$ is the number of electrons in channel $j$ of lead $\mu$. The orthonormality relation is

$$
\left\langle m_{11}, m_{12}, m_{21}, m_{22} \mid n_{11}, n_{12}, n_{21}, n_{22}\right\rangle \equiv \prod_{\mu j} \delta_{m_{\mu j}, n_{\mu j}} .
$$

Due to the Pauli exclusion principle for fermions, $n_{\mu j}=0$ or 1. Now assume a two-electron incoming state in lead 1, $|1,1,0,0\rangle$. Because of the mixing properties of the scattering process, the outgoing state is a superposition of six states with coefficients given by $\mathbf{S}$ elements. ${ }^{17}$ The first two are

$$
|1,1,0,0\rangle \text { and }|0,0,1,1\rangle \text {, }
$$

which are separable states of two electrons in one lead and the last four are

$$
|1,0,1,0\rangle, \quad|1,0,0,1\rangle, \quad|0,1,1,0\rangle, \quad \text { and }|0,1,0,1\rangle,
$$

and they can be superposed in an entangled state of one electron in each lead (orbital entanglement). The concurrence $(\mathcal{C})$ is the usual bipartite entanglement measure ${ }^{26}$ and Beenakker et $a l^{7}$ showed that, for this type of orbital entanglement, it can be written as a function of $\tau_{1}$ and $\tau_{2}$

$$
\mathcal{C}=\frac{2 \sqrt{\tau_{1}\left(1-\tau_{1}\right) \tau_{2}\left(1-\tau_{2}\right)}}{\tau_{1}+\tau_{2}-2 \tau_{1} \tau_{2}}
$$

When $\mathcal{C}=0$, the state of one electron in each lead is separable and if $\mathcal{C}=1$ then it is maximally entangled (Bell sate). If 0 $<\mathcal{C}<1$, then the state is nonseparable and partly entangled. Alternatively, entanglement formation is an entanglement cost measure related to concurrence by ${ }^{26}$

$$
\mathcal{E}(\mathcal{C})=h\left(\frac{1+\sqrt{1-\mathcal{C}^{2}}}{2}\right),
$$

where

$$
h(x)=-x \log _{2}(x)-(1-x) \log _{2}(1-x) .
$$

Of experimental interest, there is a relation between concurrence $[\mathrm{Eq} .(2)]$ and the Bell parameter related to current fluctuations. 6,7

Since the eigenvalues $\tau_{1,2}$ have statistical fluctuations due to the chaos of the system, all their functions fluctuate statistically as, for example, concurrence and entanglement formation. Therefore, these entanglement measures require a statistical treatment. Their full statistics are characterized by means of their probability densities. Using random-matrix techniques we perform a sample-to-sample numerical simulation to extract the probability densities of $\mathcal{C}$ and $\mathcal{E}$ as we explain in the next section.

\section{RANDOM-MATRIX SIMULATION}

Actually, there is no analytical method that is able to give the probability densities for concurrence and entanglement formation of a two-channel quantum dot with nonideal contacts. $^{22,27-30}$ Therefore, numerical methods are very appropriate for exploring this problem.

Let us give a brief description of a random-matrix algorithm ${ }^{25}$ applied to the chaotic quantum dot illustrated by Fig. 1. The $4 \times 4$ scattering matrix of the system [Eq. (1)] can be written as ${ }^{31}$

$$
\mathbf{S}=\mathbf{R}+\mathbf{T}\left(\mathbf{1}-\mathbf{U}_{\beta} \mathbf{R}\right)^{-1} \mathbf{U}_{\beta} \mathbf{T},
$$

where $\mathbf{U}_{1}\left(\mathbf{U}_{2}\right)$ is a random matrix which belongs to the orthogonal (unitary) circular ensemble and represents the scattering matrix of the chaotic cavity with ideal contacts. ${ }^{21}$ If $\beta=1(\beta=2)$, then there is (no) TRI in the electron dynamics inside the cavity. The matrices $\mathbf{R}$ and $\mathbf{T}$ contain information about the coefficients of reflection and transmission of the contacts

$$
\begin{gathered}
\mathbf{R}=\operatorname{diag}\left(r_{1}, r_{1}, r_{2}, r_{2}\right), \\
\mathbf{T}=\operatorname{diag}\left(t_{1}, t_{1}, t_{2}, t_{2}\right), \\
t_{\nu}=\sqrt{\Gamma_{\nu}}, \\
r_{\nu}=i \sqrt{1-\Gamma_{\nu}},
\end{gathered}
$$

where $\Gamma_{\nu}$ is the probability of electrons tunneling between lead $\nu$ and the cavity and can be viewed as the transparency of the contact varying from 0 (opaque contact) to 1 (ideal contact).

The matrix $\mathbf{U}_{\beta}$ is randomly generated following the numerical algorithm described in Ref. 32. We then use Eq. (4) to obtain $\mathbf{S}$ and, through block $\mathbf{t}$, we find the matrix $\mathbf{t t}^{\dagger}$ and its transmission eigenvalues $\tau_{1}$ and $\tau_{2}$. Therefore, the quantities $\mathcal{C}$ and $\mathcal{E}$ are obtained with Eqs. (2) and (3). By performing many iterations of this procedure, we obtain a sufficiently large sample to extract the probability densities $P(\mathcal{C})$ and $Q(\mathcal{E})$. It has already been verified that this numerical method is very efficient at producing full statistical results (probability densities) for functions of the transmission eigenvalues of a quantum dot as, for example, charge-transfer cumulants. $^{25}$

\section{RESULTS}

Using the sample-to-sample simulation described in the previous section, we obtain the distributions of concurrence and entanglement formation as the system parameters are varied: $\beta, \Gamma_{1}$, and $\Gamma_{2}$. In this section we present the results in two steps: symmetric and arbitrary contacts. All results for the opaque-contact limit $\left(\Gamma_{\nu} \rightarrow 0\right)$ were obtained by extrapolation using small values of transparency $\left(0.001 \leq \Gamma_{\nu}\right.$ 

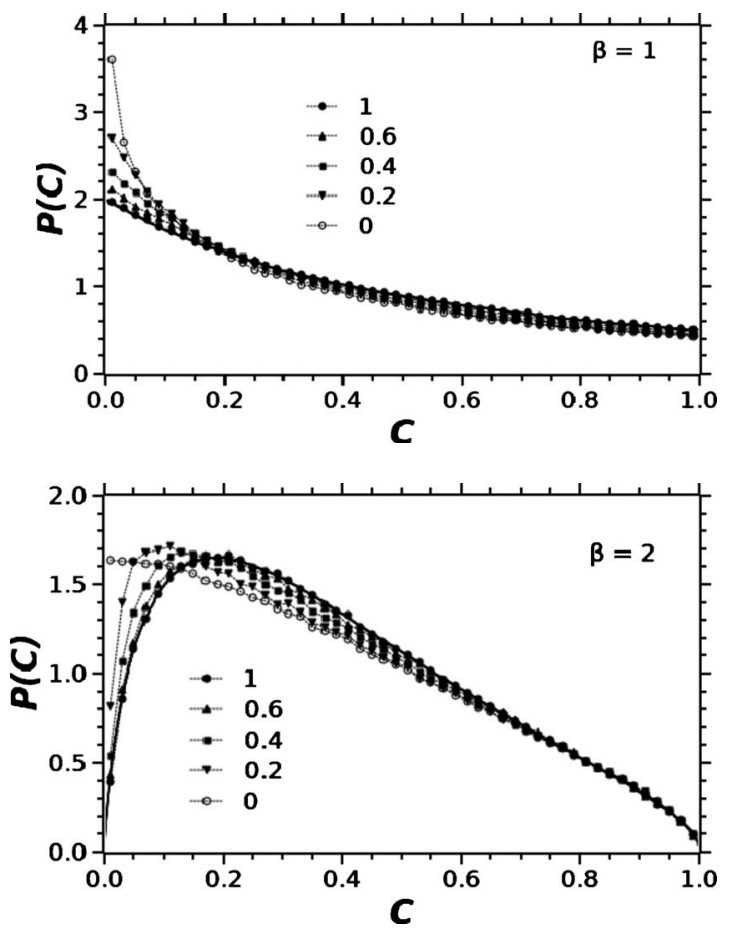

FIG. 2. Concurrence distribution for a quantum dot with symmetric contacts in the presence $(\beta=1)$ and absence $(\beta=2)$ of TRI. The transparency of the contacts is represented by the numbers labeling the symbols which are simulated data. The dotted lines are merely for guidance and the solid line is the exact result for ideal contacts (Ref. 17).

$\leq 0.006)$. Note that in this limit, the transparency is very low but it is not null. We have not obtained results for null transparency because in this situation there is no electron transport. Furthermore, we verify that our simulation produces results in agreement with the exact ones for ideal contacts. ${ }^{17}$

\section{A. Symmetric contacts}

Due to its simplicity, we start by studying the situation of symmetric contacts: $\Gamma_{1}=\Gamma_{2} \equiv \Gamma$. The concurrence distributions are illustrated in Fig. 2 for various values of $\Gamma$. Note that if $\Gamma$ decreases then $P(\mathcal{C} \ll 1)$ increases and $P(\mathcal{C} \approx 1)$ does not change significantly. Although $P(1)$ has a finite value for $\beta=1$ and is null for $\beta=2$, there are more possibilities of finding small values of $\mathcal{C}$ for $\beta=1$ than for $\beta=2$.

The distribution of entanglement formation can be observed in Fig. 3 where we note small changes when $\Gamma$ varies. Moreover, the qualitative behavior of $Q(\mathcal{E})$ is similar to that of $P(\mathcal{C})$. These two distributions indicate that the probability of finding states with weak entanglement $(\mathcal{C}, \mathcal{E} \ll 1)$ increases as the transparency of symmetric contacts decreases.

\section{B. Contacts with arbitrary transparencies}

Now we wish to study the statistics of the quantities $\mathcal{C}$ and $\mathcal{E}$ varying $\Gamma_{1}$ and $\Gamma_{2}$. Since in this situation the parameter space is very large, $\left(\Gamma_{1}, \Gamma_{2}\right) \in[0,1]^{2}$, before studying the distributions $P(\mathcal{C})$ and $Q(\mathcal{E})$ we start analyzing the averages $\langle\mathcal{C}\rangle$ and $\langle\mathcal{E}\rangle$. In Fig. 4, we can see the behavior of $\langle\mathcal{C}\rangle$ as a func-
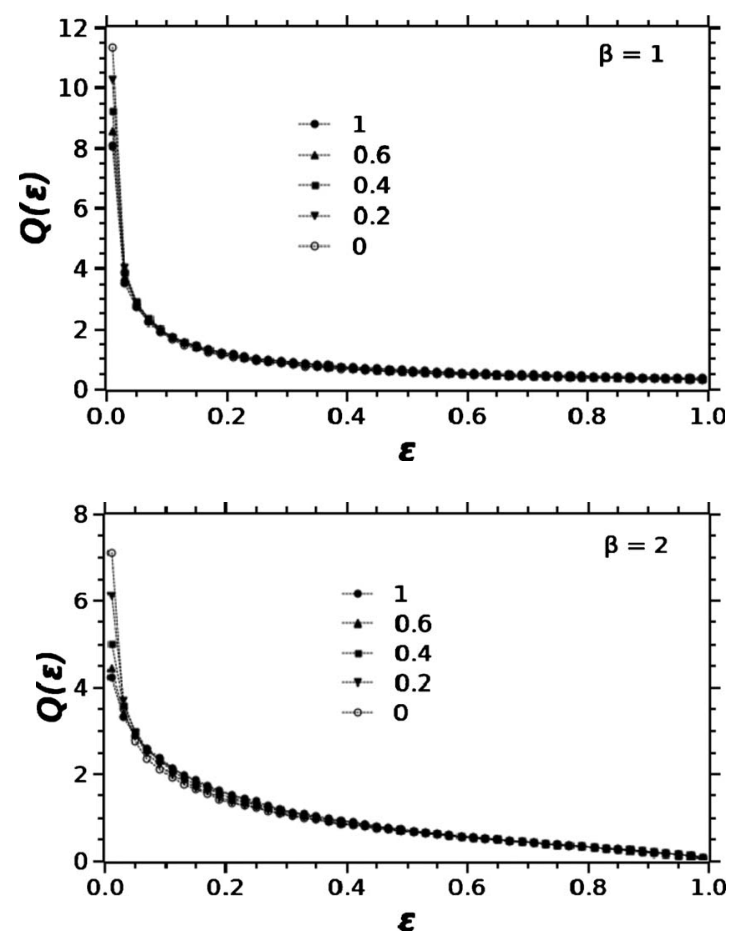

FIG. 3. Entanglement formation distribution for a quantum dot with symmetric contacts in the presence $(\beta=1)$ and absence $(\beta$ $=2$ ) of TRI. The transparency of the contacts is represented by the numbers labeling the symbols which are the simulated data. The dotted lines are merely for guidance.

tion of the transparencies. Note that the results for $\beta=1$ and 2 are very similar and if one of the contacts is opaque and the other is ideal $\left(\Gamma_{\mu}=0\right.$ and $\Gamma_{\nu}=1$ with $\left.\mu \neq \nu\right)$, then $\langle\mathcal{C}\rangle$ is maximum and it is minimum for null transparencies. Moreover, there is no significant variation in $\langle\mathcal{C}\rangle$ for $\Gamma_{1}=\Gamma_{2}$. The behavior of $\langle\mathcal{E}\rangle$ is illustrated in Fig. 5 and it is seen to be qualitatively similar to that of $\langle\mathcal{C}\rangle$. In these results, we can see that the system is invariant with respect to exchange of transparencies $\left(\Gamma_{1} \leftrightarrow \Gamma_{2}\right)$ due to transport-sense invariance.

Focusing on the optimization of orbital entanglement production in the system as a function of the parameters, the results for $\langle\mathcal{C}\rangle$ and $\langle\mathcal{E}\rangle$ suggest an investigation with extremely asymmetric contacts. Therefore, we obtain the distributions $P(\mathcal{C})$ and $Q(\mathcal{E})$ by setting $\Gamma_{1}=1$ and varying $\Gamma_{2}$. The concurrence distributions are shown in Fig. 6. Although $P(\mathcal{C})$ is weakly sensitive to transparency variation in the symmetric contact situation (see Fig. 2), note in Fig. 6 that as $\Gamma_{2}$ decreases, $P(\mathcal{C})$ becomes significantly different, because the probability of finding small values of concurrence decreases while $P(\mathcal{C} \approx 1)$ increases. Comparing the crossover from the ideal-contact situation $\left(\Gamma_{2}=1\right)$ to the opaque-contact limit $\left(\Gamma_{2} \rightarrow 0\right)$, the most probable value of concurrence $\tilde{\mathcal{C}}$ for $\beta$ $=2$ increases approximately from 0.18757 to 0.45 while for $\beta=1$ it does not change significantly, corresponding to $\tilde{\mathcal{C}}$ $\simeq 0$. The distributions of $\mathcal{E}$ are illustrated in Fig. 7 where we can observe similar qualitative behaviors between $P(\mathcal{C})$ and $Q(\mathcal{E})$. Nevertheless, note that for $\beta=2, d Q(0) / d \mathcal{E}$ is negative for $\Gamma_{2}=1$, zero for $\Gamma_{2} \approx 0.259$ and positive for $\Gamma_{2} \rightarrow 0$, and it remains negative for $\beta=1$. Moreover, the most probable 

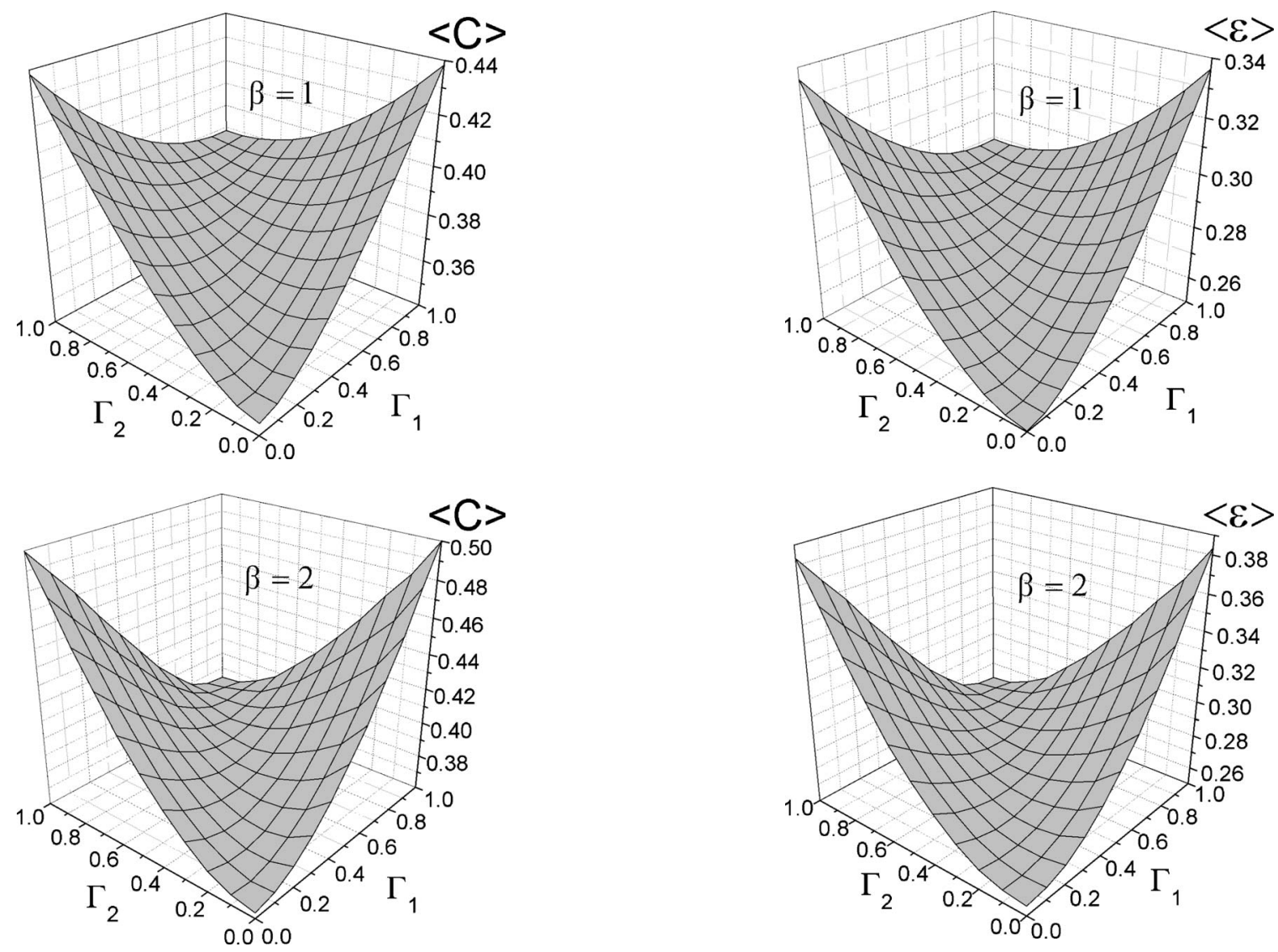

FIG. 4. Average of concurrence versus contact transparencies for a quantum dot in the presence $(\beta=1)$ and absence $(\beta=2)$ of TRI.

value of entanglement formation $\widetilde{\mathcal{E}}$ for $\beta=2$ changes from 0 , in the ideal-contact limit, to $\widetilde{\mathcal{E}} \approx 0.11$, in the opaque-contact situation, while for $\beta=1$, all values of $\Gamma_{2}$ produce $\widetilde{\mathcal{E}}=0$. Note that although the average results (see Figs. 4 and 5) are weakly sensitive to TRI effects, the distributions $P(\mathcal{C})$ and $Q(\mathcal{E})$ show distinct features for different values of $\beta$ emphasizing the comments in Ref. 17 for the ideal-contact situation.

The distributions $P(\mathcal{C})$ and $Q(\mathcal{E})$ indicate that keeping one of the contacts ideal and the other opaque confers advantages in the production of orbital entanglement, especially for $\beta$ $=2$. We investigate results for other transparency values (fixing $\Gamma_{1} \neq 1$ and varying $\Gamma_{2}$ ) where the distributions have typical behaviors for the distributions illustrated here, emphasizing that the production of orbital entanglement is optimized by increasing the asymmetry between the contacts $\left|\Gamma_{1}-\Gamma_{2}\right|$, in agreement with the average results (see Figs. 4 and 5).

Indeed, when at least one of the contacts has a very low transparency, the transport is in the tunneling regime which corresponds to $\tau_{1}, \tau_{2} \ll 1$. In this situation, the dimensionless conductance ${ }^{21,25}$ (in units of the quantum conductance $\left.2 e^{2} / h\right), g=\tau_{1}+\tau_{2}$, is too low and the transmission of charge is a rare event. Therefore, in this regime, the frequency with which entangled pairs are produced is very low. In order to approach this issue, we define the quantity $\eta_{n_{1} n_{2}}$ as the frequency with which a state occurs after the scattering, with $n_{\nu}$

FIG. 5. Average of entanglement formation versus contact transparencies for a quantum dot in the presence $(\beta=1)$ and absence $(\beta=2)$ of TRI.

electrons in lead $\nu$, and assuming that the transport is from lead 1 to lead 2. Consequently, the frequency of no transmission is $\eta_{20}=\left(1-\tau_{1}\right)\left(1-\tau_{2}\right)$, for only one electron transmitted it is $\eta_{11}=\tau_{1}\left(1-\tau_{2}\right)+\left(1-\tau_{1}\right) \tau_{2}$ and for two electrons transmitted it is $\eta_{02}=\tau_{1} \tau_{2}$. Note that $\eta_{20}+\eta_{11}+\eta_{02}=1$ as expected. In the tunneling regime, one can discard quadratic terms $\left(\tau_{1} \tau_{2}\right.$ $\approx 0$ ) yielding $\eta_{20} \approx 1-\tau_{1}-\tau_{2}=1-g, \quad \eta_{11} \approx \tau_{1}+\tau_{2}=g$, and $\eta_{02} \approx 0$. These results are quite intuitive since as the oneelectron transmission is a rare event, the simultaneous transmission of two electrons is practically impossible. Therefore, although $g \ll 1$, any charge signal detected in lead 2 corresponds to the state with one electron in each lead and with orbital entanglement degree quantified by means of the concurrence. Furthermore, if the tunneling regime is achieved through the increase in asymmetry between the contacts with no TRI, the entanglement of this detected state is optimized.

\section{SUMMARY AND CONCLUSIONS}

We have presented the probability densities of two quantifiers of entanglement, concurrence and entanglement formation, providing a full statistical description of orbital entanglement production in a chaotic quantum dot with nonideal contacts. We started by investigating the simplistic case of contacts with the same transparency (symmetric contacts) where the results show that the statistics of entangle- 

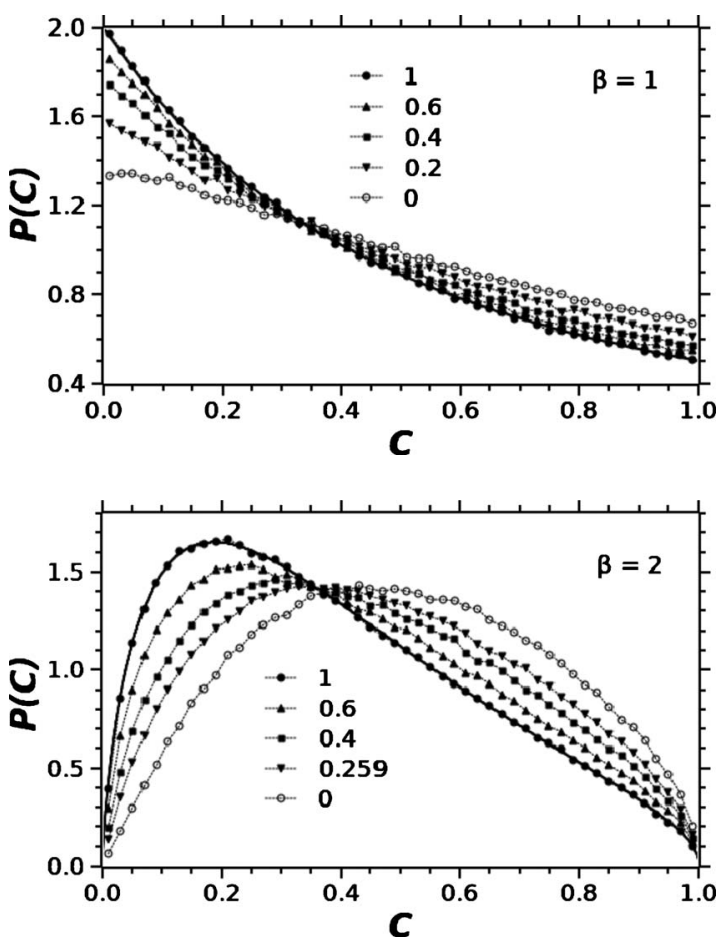

FIG. 6. Concurrence distribution for a quantum dot in the presence $(\beta=1)$ and absence $(\beta=2)$ of TRI. One of the contacts is ideal $\left(\Gamma_{1}=1\right)$ while the other has a transparency $\left(\Gamma_{2}\right)$ represented by the numbers labeling the symbols which are simulated data. The dotted lines are merely for guidance and the solid line is the exact result for ideal contacts (Ref. 17).

ment production are weakly sensitive to transparency variation and are optimized in the case of ideal contacts.

Furthermore, we have studied the more general situation of contacts with arbitrary transparency. Strong effects of TRI invariance were noted in Ref. 17 for ideal contacts. We can see that this feature is emphasized for asymmetric contacts in our results. Furthermore, we verify that as the asymmetry $\left|\Gamma_{1}-\Gamma_{2}\right|$ increases, the distributions $P(\mathcal{C})$ and $Q(\mathcal{E})$ change significantly, suggesting optimization of orbital entanglement production, especially for $\beta=2$. Therefore, orbital entanglement production is optimized when one of the contacts is ideal and the other is opaque, and when TRI is broken. The transparencies can be experimentally controlled via gate voltages $^{23}$ and the TRI can be easily broken by a magnetic field or by magnetic impurities. ${ }^{21}$

In fact, asymmetric contact effects have been observed theoretically $y^{33,34}$ and experimentally ${ }^{23}$ in electrical current fluctuations. On the other hand, these fluctuations are characterized by the transmission eigenvalues of the system. Therefore, if $\tau_{1}$ and $\tau_{2}$ are sensitive to asymmetric contact effects, then $\mathcal{C}$ and $\mathcal{E}$ (or other functions of $\tau_{1}$ and $\tau_{2}$ ) are also sensitive, in agreement with our results.
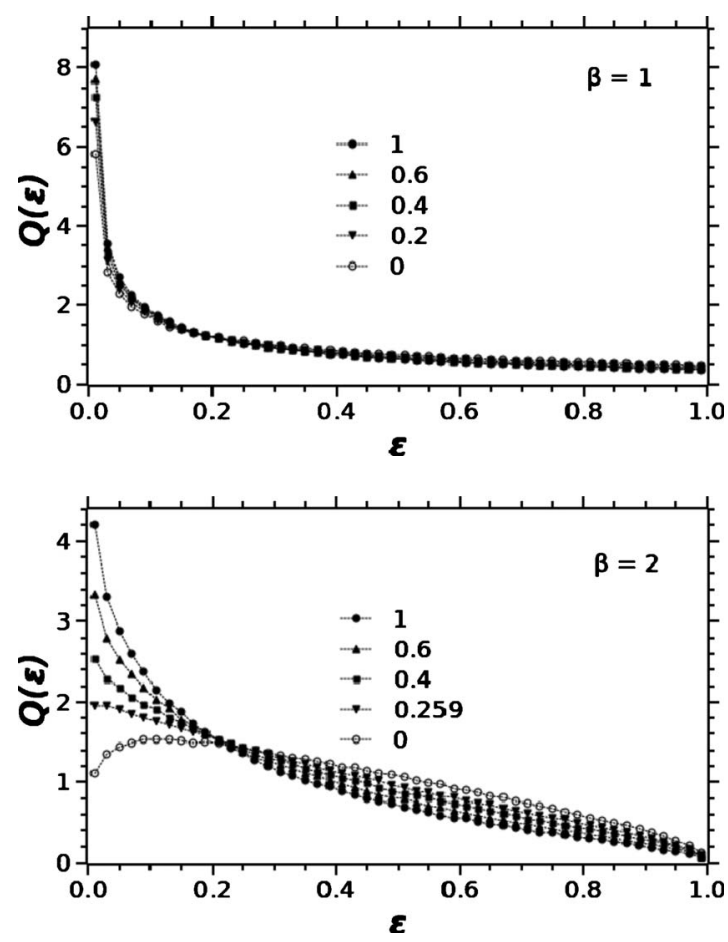

FIG. 7. Entanglement formation distribution for a quantum dot in the presence $(\beta=1)$ and absence $(\beta=2)$ of TRI. One of the contacts is ideal $\left(\Gamma_{1}=1\right)$ while the other has a transparency $\left(\Gamma_{2}\right)$ represented by the numbers labeling the symbols which are simulated data. The dotted lines are merely for guidance.

There are some difficulties in manipulating a quantum dot with extreme contact asymmetry. There is no ideal contact due to the imperfections of the system. Furthermore, when $\Gamma_{1} \approx 1$ and $\Gamma_{2} \ll 1$, the conductance of the system is very small $^{25}$ and, therefore, the electron transfer is a rare event. Thus, the crucial problem of optimizing orbital entanglement production reduces to increasing the asymmetry $\left|\Gamma_{1}-\Gamma_{2}\right|$ so that the conductance remains sufficiently large that states occur with one electron in each lead at the desired frequency. Despite these technical experimental difficulties, there is an efficient method for measuring very low currents and noise levels that was described in Ref. 23.

In future work, we can envisage the study of orbital entanglement production in this system with additional effects. For instance, the introduction of dephasing (loss of quantum coherence) can play the role of temperature effects ${ }^{24}$ and electron-electron interaction effects are very important in low-transparency contact situations due to the Coulomb blockade phenomenon. ${ }^{21,35}$

\section{ACKNOWLEDGMENT}

This work was supported by CNPq (Brazilian Agency). 
${ }^{1}$ G. Alber, T. Beth, P. Horodecki, R. Horodecki, M. Rttler, H. Weinfurter, R. Werner, and A. Zeilinger, Quantum Information (Springer, Berlin, 2001), Vol. 173.

${ }^{2}$ M. A. Nielsen and I. L. Chuang, Quantum Computation and Quantum Information (Cambridge University Press, Cambridge, 2000).

${ }^{3}$ A. M. C. Souza and F. A. G. Almeida, Phys. Rev. A 79, 052337 (2009).

${ }^{4}$ V. C. G. Oliveira, H. A. B. Santos, L. A. M. Torres, and A. M. C. Souza, Int. J. Quantum Inf. 6, 379 (2008).

${ }^{5}$ C. Anteneodo and A. M. C. Souza, J. Opt. B: Quantum Semiclassical Opt. 5, 73 (2003).

${ }^{6}$ C. W. J. Beenakker, M. Kindermann, C. M. Marcus, and A. Yacoby, in Fundamental Problems of Mesoscopic Physics, NATO Science Series II Vol. 154, edited by I. V. Lerner, B. L. Altshuler, and Y. Gefen (Kluwer, Dordrecht, 2004).

${ }^{7}$ C. W. J. Beenakker, C. Emary, M. Kindermann, and J. L. van Velsen, Phys. Rev. Lett. 91, 147901 (2003).

${ }^{8}$ P. Recher, E. V. Sukhorukov, and D. Loss, Phys. Rev. B 63, 165314 (2001).

${ }^{9}$ G. B. Lesovik, T. Martin, and G. Blatter, Eur. Phys. J. B 24, 287 (2001).

${ }^{10}$ W. D. Oliver, F. Yamaguchi, and Y. Yamamoto, Phys. Rev. Lett. 88, 037901 (2002).

${ }^{11}$ V. Bouchiat, N. Chtchelkatchev, D. Feinberg, G. B. Lesovik, T. Martin, and J. Torres, Nanotechnology 14, 77 (2003).

${ }^{12}$ D. S. Saraga and D. Loss, Phys. Rev. Lett. 90, 166803 (2003).

${ }^{13}$ S. Bose and D. Home, Phys. Rev. Lett. 88, 050401 (2002).

${ }^{14}$ A. I. Signal and U. Zlicke, Appl. Phys. Lett. 87, 102102 (2005).

${ }^{15}$ A. V. Lebedev, G. B. Lesovik, and G. Blatter, Phys. Rev. B 71, 045306 (2005).

${ }^{16}$ P. Samuelsson, E. V. Sukhorukov, and M. Büttiker, Phys. Rev. Lett. 92, 026805 (2004).

${ }^{17}$ V. A. Gopar and D. Frustaglia, Phys. Rev. B 77, 153403 (2008).

${ }^{18}$ L. Faoro, F. Taddei, and R. Fazio, Phys. Rev. B 69, 125326
(2004).

${ }^{19}$ C. W. J. Beenakker and M. Kindermann, Phys. Rev. Lett. 92, 056801 (2004).

${ }^{20}$ G. B. Lesovik, A. V. Lebedev, and G. Blatter, Phys. Rev. B 71, 125313 (2005)

${ }^{21}$ C. W. J. Beenakker, Rev. Mod. Phys. 69, 731 (1997).

${ }^{22}$ Y. V. Nazarov and Y. M. Blanter, Quantum Transport: Introduction to Nanoscience (Cambridge University Press, Cambridge, 2009).

${ }^{23}$ S. Gustavsson, R. Leturcq, B. Simovic, R. Schleser, T. Ihn, P. Studerus, K. Ensslin, D. C. Driscoll, and A. C. Gossard, Phys. Rev. Lett. 96, 076605 (2006).

${ }^{24}$ A. G. Huibers, S. R. Patel, C. M. Marcus, P. W. Brouwer, C. I. Duruöz, and J. S. Harris, Phys. Rev. Lett. 81, 1917 (1998).

${ }^{25}$ F. A. G. Almeida, S. Rodríguez-Pérez, and A. M. S. Macêdo, Phys. Rev. B 80, 125320 (2009).

${ }^{26}$ W. K. Wootters, Phys. Rev. Lett. 80, 2245 (1998).

${ }^{27}$ P. W. Brouwer and C. W. J. Beenakker, J. Math. Phys. 37, 4904 (1996).

${ }^{28}$ K. B. Efetov, Supersymmetry in Disorder and Chaos (Cambridge University Press, Cambridge, 1997).

${ }^{29}$ D. V. Savin, H.-J. Sommers, and W. Wieczorek, Phys. Rev. B 77, 125332 (2008); H.-J. Sommers, W. Wieczorek, and D. V. Savin, Acta Phys. Pol. A 112, 691 (2007).

${ }^{30}$ M. Novaes, Phys. Rev. B 75, 073304 (2007); 78, 035337 (2008).

${ }^{31}$ P. W. Brouwer, Phys. Rev. B 51, 16878 (1995).

${ }^{32}$ K. Życzkowski and M. Kuś, J. Phys. A 27, 4235 (1994).

${ }^{33}$ A. M. S. Macêdo and A. M. C. Souza, Phys. Rev. E 71, 066218 (2005).

${ }^{34}$ D. A. Bagrets and Y. V. Nazarov, Phys. Rev. B 67, 085316 (2003).

${ }^{35}$ J. N. Kupferschmidt and P. W. Brouwer, Phys. Rev. B 78, 125313 (2008). 